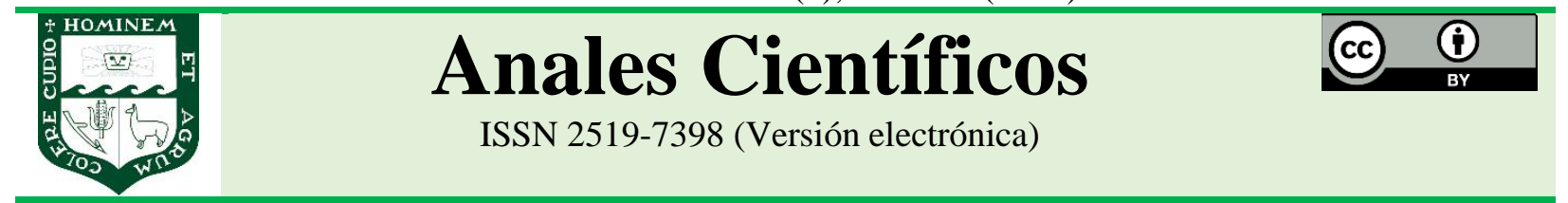

ARTÍCULO ORIGINAL - RESEARCH ARTICLE

http://dx.doi.org/10.21704/ac.v81i2.1684

\title{
SEVERIDAD DE peronospora variabilis gäum. EN CINCO VARIEDADES DE Chenopodium quinoa willd. EN CONDICIONES DE LA MOLINA
}

\section{Severity of Peronospora variabilis Gäum. in five cultivars of Chenopodium quinoa Willd. under field conditions in La Molina}

\author{
Héctor Andrés Ramírez Maguiña ${ }^{1 *}$ (D); Luz Leonor Mattos Calderón ${ }^{1}$; Alejandro Risco Mendoza ${ }^{1}$ \\ ${ }^{1}$ Facultad de Agronomía, Universidad Nacional Agraria La Molina, 15024, Lima, Perú. \\ *E-mail: amirez.ha019@gmail.com
}

Recibido: 30/06/2020; Aceptado: 15/12/2020; Publicado: 30/12/2020

\begin{abstract}
The objective of this research was to evaluate the behavior of five commercial varieties of quinoa, naturally infected by $P$. variabilis. The study was carried out at the Universidad Nacional Agraria La Molina, Lima, Peru. The varieties studied were: Amarilla de Maranganí (AMR), Pasankalla (PAS), Negra Collana (NGR), Salcedo (SAL), and Altiplano (ALT). Treatments were distributed in a complete randomized block design with five repetitions. Variables evaluated include: yield $(\mathrm{kg} / \mathrm{ha})$, plant height $(\mathrm{cm})$, disease severity using Area under Disease Progress Curve (AUDPC), protein content (\%), weight of one thousand grains ( $\mathrm{g}$ ) and grain size (\%). Comparisons of means were conducted using Tukey's test, Pearson correlation coefficient analysis and statistical significance was set at $\mathrm{p}$ $<0,05$. Results revealed statistics differences among treatments. The AMR variety had the highest yield (2628,1 $\mathrm{kg} / \mathrm{ha}$ ) followed by PAS (2201,9 kg/ha), NGR (1408,6 kg / ha), SAL (1244,4 kg / ha) and AL (649,2 kg/ha). The most susceptible variety to downy mildew was ALT (AUDPC $=4771,6)$, followed by SAL $(4337,7)$, NGR $(3796,3)$, PAS $(3377,2)$ and AMR $(2364,1)$. The correlation analysis of AUDPC showed a significant negative correlation with plant height $(r=-0,94)$, yield $(-0,88)$, and the percentage of large grains $(-0,81)$. In conclusion, AMR was the variety with the best yield and the one that showed a high tolerance to downy mildew, while ALT had the lowest yield and was the most susceptible variety.
\end{abstract}

Keywords: quinoa; Chenopodium quinoa; cultivars; downy mildew; severity; Peronospora variabilis

\section{RESUMEN}

El objetivo del presente trabajo fue estudiar el comportamiento de cinco variedades comerciales de quinua, infectadas naturalmente por $P$. variabilis en condiciones de campo. El experimento se realizó en la Universidad Nacional Agraria La Molina, Lima, Perú. Las variedades estudiadas fueron: Amarilla de Maranganí (AMR), Pasankalla (PAS), Negra Collana (NGR), Salcedo (SAL) y Altiplano (ALT). Los tratamientos tuvieron una distribución de Diseño de Bloques Completamente al Azar con cinco repeticiones. Las variables evaluadas fueron: rendimiento $(\mathrm{kg} / \mathrm{ha})$, altura de planta $(\mathrm{cm})$, severidad a través del Área Bajo la Curva de Progreso de la Enfermedad (ABCPE), contenido de proteína (\%), peso de mil granos (g) y granulometría (\%). Fueron comparadas las medias con la prueba Tukey a un nivel de significancia de 0,05 y se calculó el coeficiente correlación de Pearson. Como resultado, se observó que todas las variables estudiadas mostraron significancia. La variedad AMR tuvo el 
rendimiento más alto con 2628,1 kg/ha seguido de PAS (2201,9 kg/ha), NGR (1408,6 kg/ha), SAL (1244,4 kg/ha) y AL (649,2 kg/ha). La variedad más susceptible al mildiú fue ALT (ABCPE = 4771,6), seguido por SAL (4337,7), NGR $(3796,3)$, PAS $(3377,2)$ y AMR $(2364,1)$. El ABCPE mostró correlación inversa significativa con la altura de planta $(r=-0,94)$, con el rendimiento $(-0,88)$ y el porcentaje de granos grandes $(-0,81)$. Se concluye que, la variedad AMR fue la variedad de quinua con mejor rendimiento y la más tolerante al mildiú, en cuanto, ALT fue la variedad con menos rendimiento y la más susceptible.

Palabras clave: quinua; Chenopodium quinoa; variedades; mildiú; severidad; Peronospora variabilis

Forma de citar el artículo (Formato APA):

Ramirez, H., Mattos, L., \& Risco, A. (2020). Severidad de Peronospora variabilis Gäum. en cinco variedades de Chenopodium quinoa Willd. en condiciones de La Molina. Anales Científicos. 81(2), 395-404. http://dx.doi.org/10.21704/ac.v81i2.1684

Autor de correspondencia (*): Héctor Andrés Ramírez Maguiña. Email: amirez.ha019@ gmail.com

(C) Los autores. Publicado por la Universidad Nacional Agraria La Molina.

This is an open access article under the CC BY

\section{INTRODUCCIÓN}

La quinua (Chenopodium quioa Willd.) es un cultivo que, desde su domesticación, ha jugado un rol importante en la subsistencia de las sociedades andinas (Romero, 2015). En la última década, su producción y consumo han aumentado aceleradamente debido a la revalorización de las propiedades nutritivas de sus granos y por ser un cultivo adaptable a condiciones de estrés salino y de sequía (Bazile et al., 2016; Romero, 2017).

Como parte del manejo agronómico de la quinua, se deben tener en cuenta el control de los organismos causantes de enfermedades. Entre ellos se mencionan a Rhizoctonia sp., Fusarium sp., Phoma exigua, Phoma sp., Ascochyta hyalospora, Cladosporium sp. (Cruces, 2016; Gómez-Pando \& Aguilar, 2016). Sin embargo, el mildiú, ocasionado por el oomyceto Peronospora variabilis, es considerada la enfermedad más importante (Aguilar \& Ibañez-Tremolada, 2019).

Peronospora variabilis Gaüm. ha sido identificado y detectado infectando a la quinua, mediante estudios morfológicos, histológicos y moleculares (Choi et al., 2010; El-Assiuty et al., 2019a; El-Assiuty et al., 2019b; Taha, 2020). Los oomycetos causantes de los "mildiús" pertenecen a la familia Peronosporaceae y se caracterizan por ser parásitos obligados con un rango de hospedante altamente específico (Thines \& Choi, 2016). Los síntomas de esta enfermedad son manchas cloróticas con áreas necróticas centrales en las hojas y su posterior defoliación, lo cual disminuye el área fotosintética de la planta y su capacidad productiva (Cruces, 2016).

Los programas de mejoramiento genético de la quinua, en la región andina, han dado especial atención al germoplasma resistente al mildiú (Gómez-Pando, 2015; Gómez-Pando et al., 2019). En este contexto, es necesario el estudio de la gran diversidad genética existente de quinua en el Perú, como centro de origen, con el fin de identificar germoplasma con resistencia al mildiú y así ser usado en programas de mejoramiento genético y cómo método de control dentro de un programa integrado, en zonas de alta incidencia de la enfermedad. Es por ello, que el presente trabajo de investigación planteó como objetivo, estudiar el comportamiento de cinco variedades comerciales de quinua (Chenopodium quinoa), infectadas naturalmente por $P$. variabilis en condiciones de campo.

\section{MATERIALES Y MÉTODOS}

\section{Ubicación del experimento y condiciones meteorológicas}

El trabajo experimental fue realizado en un campo experimental del Departamento de Fitopatología de Universidad Nacional Agraria La Molina, Lima, Perú, entre los meses de julio y diciembre del 2018. Los datos meteorológicos se muestran en la Tabla 1.

\section{Material biológico}

En la Tabla 2 se muestran las variedades de quinua empleadas y sus respectivas características. Dos semanas antes del inicio del trabajo de investigación (25 
de Julio del 2018), se sembraron dos hileras de quinua de la variedad INIA Salcedo, en los bordes del campo experimental. Dichas plantas se dejaron infectar naturalmente por $P$. variabilis. De esta forma, se promovió una infección uniforme en todas las parcelas que fueron sembradas posteriormente. Se eligió la variedad Salcedo, debido su alta susceptibilidad al mildiú según previas experiencias.

\section{Manejo del cultivo y diseño experimental}

Los cinco tratamientos (variedades) fueron sembras dos semanas después de la siembra del cerco vivo (08 de agosto del 2018), se dispusieron en un Diseño de Bloques Completamente al Azar (DBCA), con cinco repeticiones (Figura 1). Cada bloque estuvo constituido por plantas de la misma variedad sembradas a chorro contínuo y a doble hilera en ocho metros, a lo largo de dos cintas de riego. El distanciamiento entre hileras fue de $45 \mathrm{~cm}$, y entre parcelas del mismo bloque y las laterales de $1 \mathrm{~m}$, con 30 plantas por metro lineal (luego del desahije).

Para evitar la muerte prematura de las plantas, se realizó una aplicación foliar de fungicida contra el mildiú a los 18 días después de la siembre (DDS), con el ingrediente activo Metalaxil (a concentración de 35\%) a una dosis de $1 \mathrm{~g} / \mathrm{l}$.

Para el control de plagas, se realizaron aplicaciones de insecticidas a dosis comercial, con los ingredientes activos Fipronil, Imidaclopid, Methomyl según la presencia de gusanos comedores de hojas y grano (Spodoptera sp., Eurysacca sp., Chloridea sp.), áfidos y chinches.

El total de fertilizante aplicado durante la campaña fue $149 \mathrm{~kg} / \mathrm{ha}$ de nitrógeno, $129 \mathrm{~kg} / \mathrm{ha}$ de fósforo y 136 $\mathrm{kg} / \mathrm{ha}$ de potasio. Se inyectó mediante un tubo Venturi en el cabezal de riego, fraccionado en dosis semanales hasta los 100 DDS.

\section{Cosecha y trilla}

La cosecha de las variedades Salcedo, Pasankalla y Altiplano se realizó a los 123 DDS; mientras que la cosecha de las variedades Amarilla de Maranganí y Negra Collana se realizó a los 148 DDS. Las inflorescencias fueron cortadas y secadas en el campo, las cuales posteriormente fueron trilladas con una trilladora estacionaria mecánica y venteadas hasta dejar el grano limpio sin residuos.

Tabla 1. Promedios mensuales de datos meteorológicos de La Molina, Lima, año 2018.

\begin{tabular}{lccccc}
\hline \multicolumn{1}{c}{ Mes } & $\begin{array}{c}\text { Temperatura } \\
\text { máxima }\left({ }^{\circ} \mathbf{C}\right)\end{array}$ & $\begin{array}{c}\text { Temperatura } \\
\text { mínima }\left({ }^{\circ} \mathbf{C}\right)\end{array}$ & $\begin{array}{c}\text { Temperatura } \\
\text { promedio }\left({ }^{\circ} \mathbf{C}\right)\end{array}$ & $\begin{array}{c}\text { Humedad relativa } \\
\text { promedio }(\boldsymbol{\%})\end{array}$ & $\begin{array}{c}\text { Lluvia } \\
(\mathbf{m m})\end{array}$ \\
\hline Junio & 16,2 & 27,9 & 14,1 & 84,6 & 0,20 \\
Julio & 16,2 & 27,9 & 14,3 & 83,7 & 0,25 \\
Agosto & 15,9 & 27,9 & 13,7 & 81,7 & 0,07 \\
Septiembre & 16,7 & 27,9 & 13,8 & 79,7 & 0,04 \\
Octubre & 17,8 & 28,0 & 15,0 & 78,4 & 0,63 \\
Noviembre & 19,2 & 27,9 & 15,6 & 75,6 & 0,02 \\
Diciembre & 21,0 & 27,5 & 17,1 & 74,6 & 0,01 \\
Promedio & 17,6 & 27,9 & 14,8 & 79,8 & 0,2 \\
\hline
\end{tabular}

Elaborado con datos de la Estación Meteorológica von Humboldt (OVH), La Molina, Lima-Perú.

Tabla 2. Datos del material genético empleado.

\begin{tabular}{lcccc}
\hline \multicolumn{1}{c}{ Variedad } & EEA de producción & Lugar de procedencia & Categoría de semilla & Ecotipo \\
\hline Amarilla de Maranganí & EEA Andenes & Cusco & Autorizada & Valle \\
Altiplano & EEA Illpa & Puno & Autorizada & Altiplano \\
INIA Salcedo & EEA Illpa & Puno & Autorizada & Altiplano \\
Negra Collana & EEA Illpa & Puno & Autorizada & Altiplano \\
Pasankalla & EEA Illpa & Puno & Altiplano \\
\hline
\end{tabular}

EEA: Estación Experimental Agraria del Instituto de Innovación Agraria. 


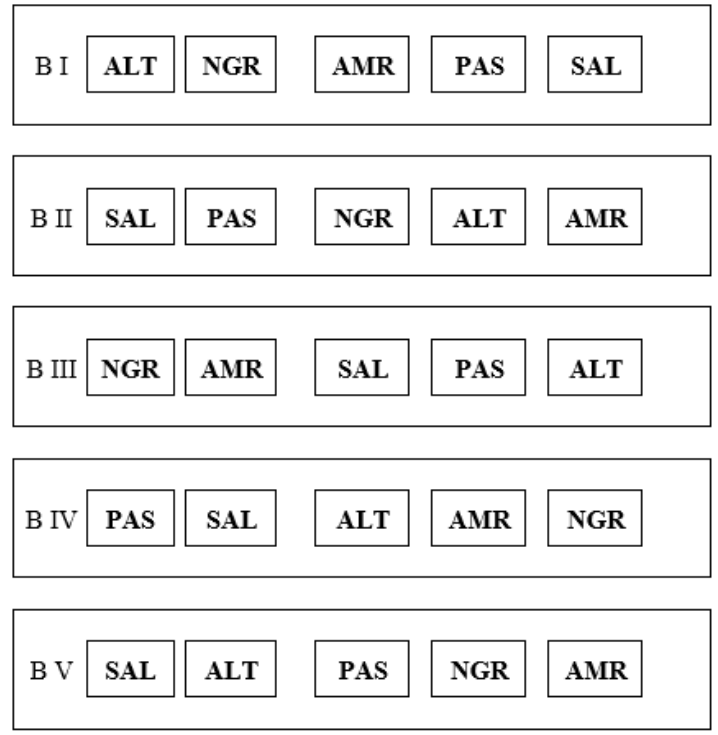

Figura 1. Plano del campo experimental y distribución de los tratamientos por cada bloque.

ALT: Altiplano; NGR: Negra Collana; AMR: Amarilla de Maranganí; PAS: Pasankalla; SAL: Salcedo; B: Bloque; números romanos (repetición).

\section{Evaluación de la enfermedad}

Se marcaron 20 plantas al azar en cada parcela, las cuales fueron evaluadas a lo largo de toda la campaña. Para ello, se usó el método descrito por Risco y Mattos (2015), el cual consistió en estimar visualmente el porcentaje de área foliar de la planta entera con síntomas de mildiú, con valores entre el 0\% y $100 \%$, ya que según Risco et al. (2018), se ha demostrado que este método proporciona resultados más precisos.

En total, se hicieron 13 evaluaciones, cada 4 a 6 días, iniciando a los 45 DDS y hasta los 100 DDS, con los datos obtenidos, se elaboraron las curvas de progreso de la enfermedad y se calculó el Área Bajo la Curva de Progreso de la Enfermedad (ABCPE), para cada parcela, usando la siguiente fórmula:

$$
A B C P E=\sum_{i=1}^{n-1} \frac{\left(y_{i}+y_{i+1}\right)}{2}\left(t_{i+1}-t_{i}\right)
$$

donde “ $n$ " es el número de evaluaciones, " $y$ ” es la severidad y " $t$ ” el número de días después de la siembra. Se consideró como punto de inicio $(\mathrm{t}, \mathrm{y})=$ $(0,0)$.

\section{Variables agronómicas}

El rendimiento $(\mathrm{kg} / \mathrm{ha})$ se calculó pesando el total de granos limpios producidos por cada unidad experimental luego del proceso de trilla y venteo, por otra parte, la altura de planta $(\mathrm{cm})$ se halló midiendo las plantas marcadas con una cinta métrica, desde la base de la planta hasta la punta de la panoja principal, un día antes que fueran cosechadas.

\section{Variables de calidad de grano}

Para el análisis de calidad, se tomaron muestras de $30 \mathrm{~g}$ de quinua por cada parcela, se retiraron los pericarpios excedentes y se pasaron por un tamiz de $1,18 \mathrm{~mm}$ de apertura para remover las impurezas y granos vanos.

Para hallar el peso de mil granos (en gramos), se contaron mil granos de cada muestra y se pesaron con una balanza electrónica Mettler Toledo. Para la granulometría, se pesaron $10 \mathrm{~g}$ de cada muestra y se separaron usando zarandas vibratorias durante 3 minutos, con diámetros de apertura de $1,7 \mathrm{~mm}$ y 1,4 $\mathrm{mm}$, separando los granos que lograron pasar cada una de las zarandas. Los datos se expresaron en porcentaje de peso de granos mayores a $1,7 \mathrm{~mm}$, de entre 1,7 y 1,4 mm y menores a 1,4 $\mathrm{mm}$ (estos dos últimos solo se usaron para el análisis de correlación). Para hallar el porcentaje de proteína se usó el equipo Infratec 1241 Grain Analyser, siguiendo los protocolos del laboratorio de calidad, del Programa de Cereales y Granos Andinos de la UNALM.

\section{Análisis estadísticos}

Para el análisis estadístico, se usó el programa R v.3.6.1 mediante la interfaz RStudio. Si inició el análisis, verificando el supuesto de normalidad de los errores experimentales con el test de ShapiroWilk y también fue verificado la homogeneidad de varianzas de los tratamientos con el test de Levene, a un nivel de significancia de 0,05, usando el paquete estadístico "car". Seguidamente, fue realizado el análisis de variancia (ANVA) para después comparar las medias de los valores de cada variable, 
a través la prueba de Tukey con 0,05 de significancia, usando el paquete estadístico "agricolae". Finalmente, fueron obtenidos los valores de coeficientes de correlación de Pearson, correlacionando los valores del ABCPE con los valores medios de las demás variables evaluadas.

\section{RESULTADOS Y DISCUSIÓN}

\section{Severidad de la infección de mildiú}

La enfermedad se detectó, en el campo experimental, en uno de los cercos vivos a los 5 DDS de los tratamientos y a los 16 DDS, todas las parcelas experimentales mostraron plantas con síntomas de infección, por lo que se optó por controlar la enfermedad mediante una aplicación química, como ya se detalló en la metodología.

Las curvas de progreso de la enfermedad de cada variedad mostraron un patrón de desarrollo similar, pero a velocidad diferente (Figura 2). La disminución, observada entre los 57 y 62 DDS, se debió al rápido crecimiento vegetativo de las plantas en dicha etapa, similar a los resultados de (Danielsen \& Munk, 2004). Luego de ello, la enfermedad se incrementó en todos los tratamientos, tendiendo al $100 \%$, en la mayoría de las variedades, a excepción de la variedad Amarilla de Maranganí, en la cual la enfermedad se retardó mucho más. En cuanto a la variedad Negra Collana, esta llegó a menos del $90 \%$ de severidad debido al crecimiento de los brotes basales de la planta como respuesta a la defoliación ocasionadas por $P$. variabilis, según lo observado el comportamiento de esta variedad.

El análisis de variancia mostró diferencias estadísticas entre los tratamientos del ABCPE. La prueba de Tukey $(\alpha=0,05)$ clasificó a cada variedad en un grupo estadístico diferente (Tabla 3).

La variedad Amarilla de Maranganí obtuvo el ABCPE más bajo $(2364,1)$, con una diferencia estadística notable con los demás tratamientos, seguido por la variedad Pasankalla que resultó con un valor ABCPE de 3377,2 y comparando con la curva del progreso de la enfermedad, estas dos variedades son las que mostraron menor desarrollo de la enfermedad en el tiempo que duró el experimento; esto ratifica lo descrito por algunos autores que indican que la quinua de ecotipo de valle, como la Amarilla de Maranganí, poseen más resistencia de tipo cuantitativa al mildiú, en comparación con las de altiplano (Gabriel et al., 2012; Gómez-Pando \& Aguilar, 2016). Esto se debe, en términos evolutivos, a que las quinuas de valle se domesticaron y cultivaron en condiciones ambientales favorables para el mildiú, por lo que solo las más resistentes perduraron en el tiempo (Bonifacio, 2001).

Por otra parte, las demás variedades mostraron diferencias significativas para el ABCPE con valores más altos, Negra Collana (3796,3), Saceldo $(4337,7)$ y Altiplano $(4771,6)$, mostrando mayor susceptibilidad al mildiú, pudiendo deberse a la alta variabilidad genética que poseen los diferentes grupos de quinuas (Gómez-Pando \& Aguilar, 2016), e incluso a la gran diversidad en la susceptibilidad al mildiú de la quinua, inclusive entre genotipos del mismo eco tipo (Kumar et al., 2006; Gabriel et al., 2012; Khalifa \& Thabet, 2018). Adicionalmente, Danielsen y Munk (2004), demostraron que la variedad Amarilla de Maranganí fue una de las más resistentes y la INIA Salcedo una de las más susceptibles a $P$. variabilis, agente causal del mildiú de la quinua.

Es importante señalar que todas las variedades usadas en este trabajo de investigación están clasificadas como "tolerantes" o "moderadamente tolerantes" al mildiú en diferentes guías de cultivo de quinua (Estrada, 2013; Gómez-Pando \& Aguilar, 2016) y en el catálogo de variedades oficial del Instituto Nacional de Innovación Agraria (INIA) (Apaza et al., 2013). Los resultados obtenidos en este trabajo mostraron que existe mayor diversidad en los niveles de expresión de síntomas entre las variedades comerciales (Figura 3 y 4).

\section{Variables agronómicas}

El análisis de varianza $(\alpha=0,05)$ indica diferencias estadísticas entre los tratamientos para las variables de rendimiento y altura de planta. 


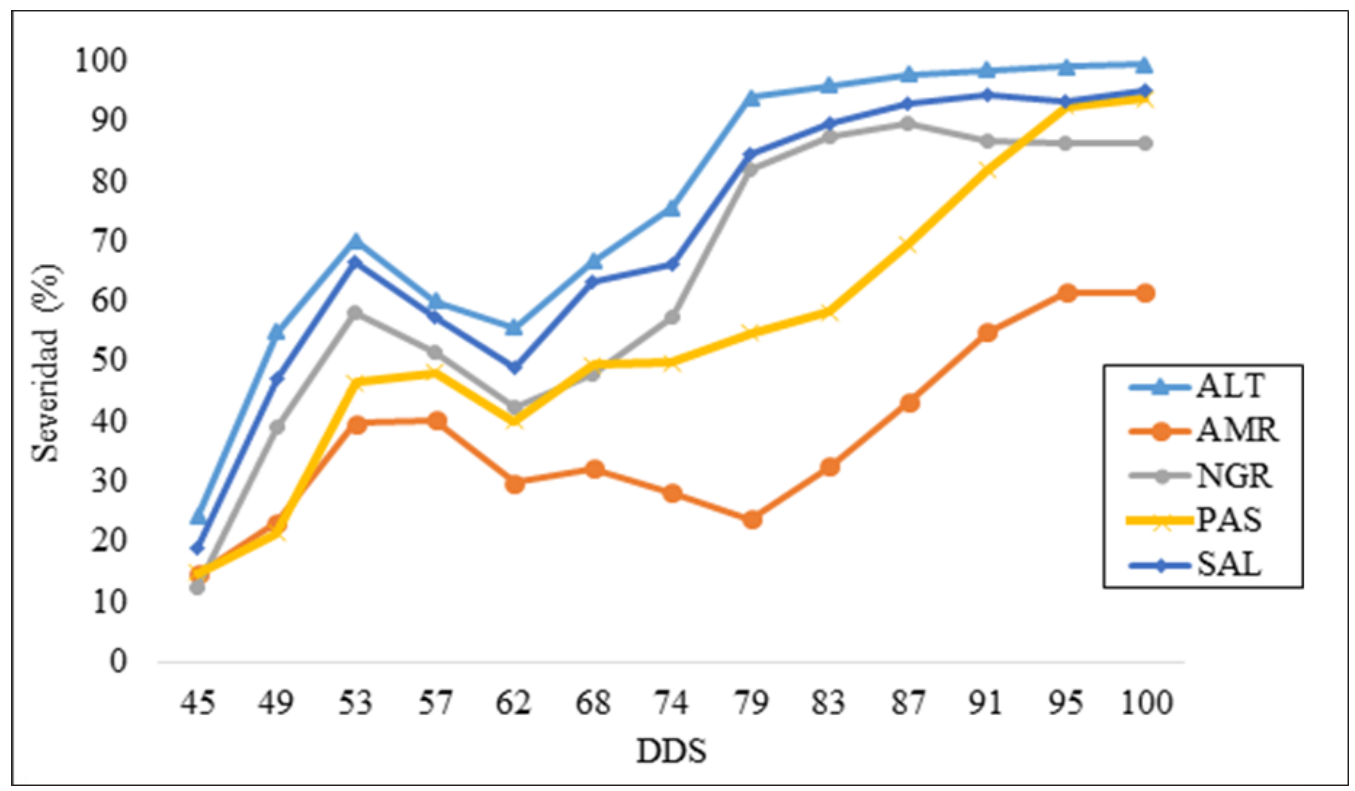

Figura 2. Curva del progreso del mildiú de la quinua (P. variabilis) en cinco variedades diferentes. La Molina, 2018. ALT: Altiplano; AMR: Amansiderilla de Maranganí; NGR: Negra Collana; PAS: Pasankalla; SAL: Salcedo; DDS: Días después de la siembra

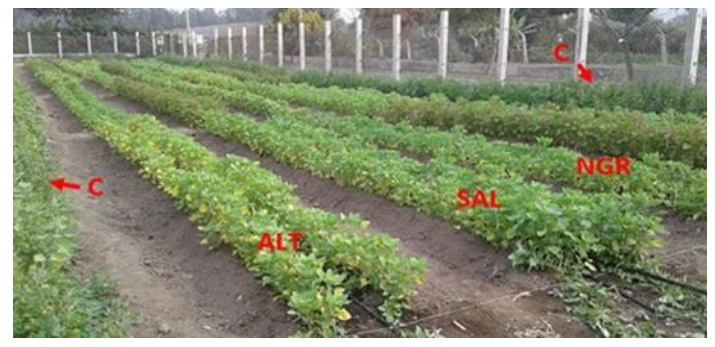

Figura 3. Campo experimental de Fitopatología de la UNALM, a 51 DDS. C: cerco vivo. ALT: variedad Altiplano. SAL: variedad Salcedo. NGR: variedad Negra Collana.

Tabla 3. Comparación de medias de las variables evaluadas en cinco variedades de quinua.

\begin{tabular}{|c|c|c|c|c|c|c|c|c|c|c|c|c|c|c|}
\hline \multirow{3}{*}{$\begin{array}{l}\text { Variedad } \\
\text { Negra } \\
\text { Collana }\end{array}$} & \multirow{2}{*}{\multicolumn{2}{|c|}{ ABCPE $^{*}$}} & \multirow{2}{*}{\multicolumn{2}{|c|}{$\begin{array}{c}\text { Rendimiento } \\
\text { (kg/ha) }\end{array}$}} & \multirow{2}{*}{\multicolumn{2}{|c|}{$\begin{array}{l}\text { Altura } \\
(\mathbf{c m})\end{array}$}} & \multirow{2}{*}{\multicolumn{2}{|c|}{$\begin{array}{c}\text { Peso de } \\
\text { mil } \\
\text { granos (g) }\end{array}$}} & \multicolumn{4}{|c|}{ Granulometría (\%) } & \multirow{2}{*}{\multicolumn{2}{|c|}{$\begin{array}{l}\text { Contenido } \\
\text { de proteína } \\
(\%)\end{array}$}} \\
\hline & & & & & & & & & \multicolumn{2}{|c|}{$\begin{array}{l}\text { Más de } \\
1,7 \mathrm{~mm}\end{array}$} & \multirow{2}{*}{$\begin{array}{c}\text { Entre 1,7 } \\
\text { y } \mathbf{1 , 4} \mathbf{~ m m} \\
56,8\end{array}$} & \multirow{2}{*}{$\begin{array}{c}\text { Menos de } \\
\mathbf{1 , 4} \mathbf{~ m m}\end{array}$} & & \\
\hline & 3796,3 & $\mathrm{c}$ & 1408,6 & $\mathrm{~b}$ & 94,5 & $\mathrm{c}$ & 2,085 & $\mathrm{~d}$ & 1,0 & d & & & 13,2 & $\mathrm{c}$ \\
\hline Altiplano & 4771,6 & $\mathrm{e}$ & 649,2 & $\mathrm{c}$ & 83,6 & $\mathrm{c}$ & 2,795 & $\mathrm{c}$ & 11,7 & $\mathrm{c}$ & 71,4 & 16,9 & 15,3 & $\mathrm{a}$ \\
\hline Salcedo & 4337,7 & $\mathrm{~d}$ & 1244,4 & $\mathrm{bc}$ & 87,1 & c & 2,696 & $\mathrm{c}$ & 12,0 & $\mathrm{c}$ & 71,8 & 16,2 & 14,9 & $\mathrm{ab}$ \\
\hline Pasankalla & 3377,2 & $\mathrm{~b}$ & 2201,9 & $\mathrm{a}$ & 117,1 & $\mathrm{~b}$ & 3,210 & $\mathrm{~b}$ & 28,7 & $\mathrm{~b}$ & 64,0 & 7,2 & 12,9 & $\mathrm{c}$ \\
\hline $\begin{array}{l}\text { Amarilla de } \\
\text { Maranganí }\end{array}$ & 2364,1 & $\mathrm{a}$ & 2628,1 & $\mathrm{a}$ & 145,3 & $\mathrm{a}$ & 3,592 & a & 55,1 & a & 40,4 & 4,4 & 14,3 & $\mathrm{~b}$ \\
\hline
\end{tabular}

*Valores seguidos de la misma letra, en la misma columna, no difieren estadísticamente según la prueba de Tukey ( $\alpha=$ $0,05)$. 


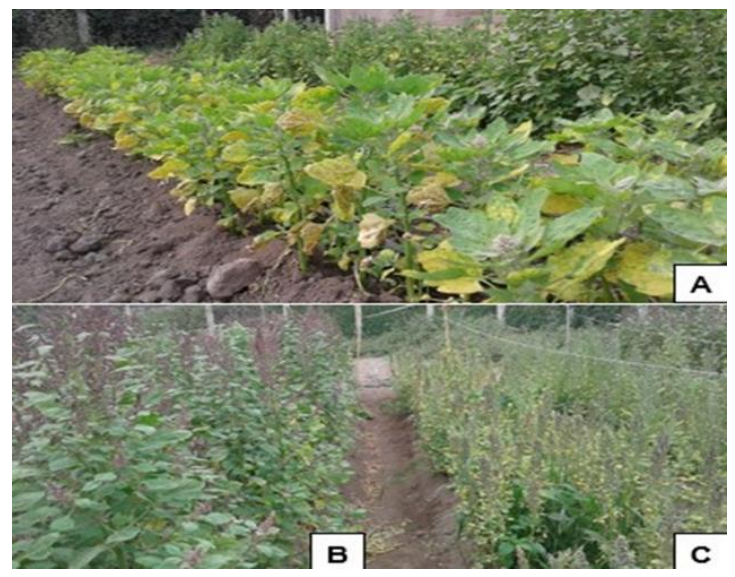

Figura 4. Campo experimental de Fitopatología de la UNALM. (A) variedad Altiplano a 51 DDS con sintomatología típica del mildiú causado por P. variabilis: hojas con manchas cloróticas y esporulación gris por el envés de las hojas. (B) variedad Amarilla de Maranganí con 81 DDS (B) y variedad Altiplano a 81 DDS y (C) variedad Altiplano a 81 DDS. Nótese la diferencia en vigor y nivel de infección entre estas dos variedades.

Altiplano fue la variedad de quinua con menor rendimiento $(649,2 \mathrm{~kg} / \mathrm{ha})$, significativamente diferente a las variedades Amarilla de Maranganí $(2628,1 \mathrm{~kg} / \mathrm{ha})$ y Pasankalla $(2201,9 \mathrm{~kg} / \mathrm{ha})$ que fueron las de mejor rendimiento. Esta variedad Altiplano, también fue diferente de Negra Collana (1408,6 kg/ha) e igual a la variedad Salcedo, que obtuvo 1244,4 kg/ha (Tabla 3).

Algunos de los autores indican que, los rendimientos de la quinua varían entre variedades y ecotipos, ya que existen diferencias en su capacidad genética de producción e interacción entre ésta, el medio ambiente y manejo agronómico (Mujica et al., 2001; Marca et al., 2015). Por otra parte, Danielsen y Munk (2004), en su investigación, obtuvieron resultados similares a los de este estudio, en cuanto al valor de los rendimientos de las variedades Amarilla de Maranganí y Salcedo.

Como parte del medio ambiente y manejo agronómico, puede ser considerado en nivel tecnológico de conducción del cultivo. En este caso, el nivel de tecnología que se empleó puede ser considerado de nivel medio a alto, debido al uso de sistema de riego por goteo, uso de fertilizantes y uso de productos químicos para el control de plagas. Sin embargo, los rendimientos fueron menores de lo esperado. Gómez-Pando y Aguilar (2016), indican que el rendimiento de la quinua puede variar entre 1 a 7 toneladas por hectárea, dependiendo de los factores suelo, clima y tecnología empleada.

En cuanto a la altura de planta, la variedad Amarilla de Maranganí (145,3 cm) fue la de mayor tamaño y difiere estadísticamente respecto a las otras variedades, con una diferencia considerable a la variedad que le sigue en tamaño, la Pasankalla $(117,1 \mathrm{~cm})$. Por otro lado, las alturas de las variedades Negra Collana, Salcedo y Altiplano no difirieron estadísticamente.

En relación con los resultados obtenidos en esta investigación Gómez-Pando y Eguiluz (2011), indican que la altura es un rasgo que también está determinado, en gran medida, por el ecotipo de la variedad y que las quinuas de valle se caracterizan por la predominancia de materiales de mayor altura. De igual manera Gómez-Pando y Aguilar (2016) mencionan que las quinuas de valle pueden alcanzar y sobrepasar los 2,4 m de altura; mientras que, las de altiplano promedian entren los 0,5 y $1,5 \mathrm{~m}$ de altura.

\section{Variables de calidad de grano}

El análisis de varianza $(\alpha=0,05)$ indica diferencias estadísticas entre los tratamientos para las variables de peso de mil granos, porcentaje de granos de más de $1,7 \mathrm{~mm}$ y contenido de proteína.

De todas las variedades de quinua, la Amarilla de Maranganí fue la de mayor peso de mil granos $(3,592 \mathrm{~g})$ y la Negra Collana fue la de menor $(2,085$ g), mostrando una diferencia estadística con los otros tratamientos, donde los pesos de las variedades Altiplano (2,795 g) y Salcedo (2,696 g) se agruparon en el mismo grupo de homogeneidad (Tabla 3). Gabriel et al. (2012), mostró una tendencia semejante en estos valores.

Según la Norma Técnica Peruana de la quinua INDECOPI (2009), los granos se pueden clasificar en cuanto a su tamaño según el porcentaje de granos que quedan retenidos entre los tamices de 1,7 y 1,4 $\mathrm{mm}$, siempre y cuando pasen el $85 \%$ en una 
determinada medida. Dado estas cifras, el porcentaje de granos considerados de buena calidad de este ensayo, no se podrían clasificar según los parámetros de las normas de INDECOPI, puesto que, el porcentaje de granos de más de $1,7 \mathrm{~mm}$ en la variedad Amarilla de Maranganí fue de 55,1\%, siendo este el valor más alto y estadísticamente diferente al resto, seguido por la variedad Pasankalla, Salcedo, Altiplano (estas dos últimas sin diferencia estadística) y finalmente la variedad Negra Collana (Tabla 3).

En cuanto al contenido de proteína, la variedad con mayor valor fue la Altiplano (15,3\%), seguida de la Salcedo (14,9\%), las cuales no tuvieron diferencia estadística. Las variedades con menor contenido de proteína, la Negra Collana $(13,2 \%)$ y Pasankalla $(12,9 \%)$, resultaron en un grupo de homogeneidad diferente al resto (Tabla 3). Los resultados concuerdan con el contenido de proteína promedio de la quinua de entre 13,6\% a 16,7\% (Navruz-Varli \& Sanlier, 2016; Vilcacundo \& HernándezLedesma, 2017).

\section{Análisis de correlación}

El coeficiente de Pearson indicó que la severidad del mildiú en términos de ABCPE tuvieron una alta correlación inversa significativa $(r>|0,8|)$ con las variables, altura de planta, rendimiento, porcentaje de granos de más de $1,7 \mathrm{~mm}$ y el porcentaje de granos de entre 1,7 y 1,4 mm (Tabla 4) y una correlación positiva con granulometría $(r=0,84)$. Con estas relaciones, claramente se demuestra que la intensidad de la enfermedad del mildiú en el cultivo de quinua influye de manera negativa a la altura de planta, porcentaje de granos mayores y, por ende, el rendimiento. Por otro lado, hay un efecto positivo en el porcentaje de granos menores, es decir, disminuye la calidad en términos de calibre.

En concordancia con estos resultados, Danielsen y Munk (2004), encontraron alta correlación negativa entre el rendimiento y el ABCPE. Gabriel et al. (2012), también hallaron correlación negativa entre la ABCPE con el rendimiento y altura de planta; sin embargo, hallaron correlación positiva con el peso de cien granos. Adicionalmente, se ha comprobado que el mildiú puede causar reducción de la altura de planta en cultivos como girasol y pepinillo (Bán et al., 2004; Mohamed et al., 2016). Por otro lado, Risco y Mattos (2015), no encontraron diferencias significativas en el peso de mil granos, granulometría ni contenido de proteína en parcelas de quinua con diferente severidad de mildiú.

Tabla 4. Coeficientes correlación de Pearson (r) del ABCPE con las variables agronómicas y de calidad de grano.

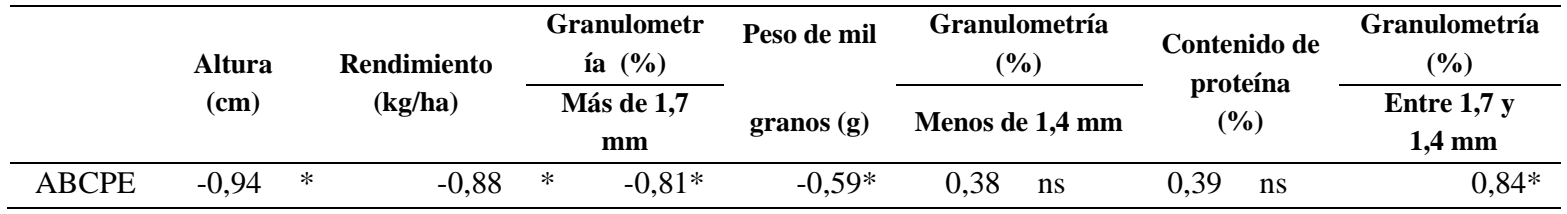

$*$ Significancia a un nivel de 0,05 , ns: no significativo a un nivel de 0,05 .

\section{CONCLUSIONES}

Dentro de las cinco variedades comerciales de quinua que fueron infectadas naturalmente por $P$. variabilis en condiciones de campo, Amarilla de Maranganí fue la que tuvo mejorcomportamiento en termino de, rendimientomás alto, más tolerante al mildiú, mayordesarrolló de altura de planta, el más alto enpeso de mil granos y con el porcentaje más alto de granos grandes. En contraparte, la variedad
Altiplano, tuvo un comportamiento menos favorable, con el rendimiento más bajo, la más susceptible a $P$. variabilis y la fue la que desarrolló menos altura de planta.

\section{Agradecimientos}

El autor por correspondencia expresa su agradecimiento a los profesores de la Facultad de Agronomía de la Universidad Nacional Agraria La Molina, Carlos Cadenas, Andrés Casas, Jorge Jiménez, José Falconi, al Programa de Cereales y 
Granos nativos y a las personas que apoyaron durante la fase experimental.

\section{Conflictos de intereses}

Los autores firmantes del presente trabajo de investigación declaran no tener ningún potencial conflicto de interés personal o económico con otras personas $\mathrm{u}$ organizaciones que puedan influir indebidamente con el presente manuscrito.

\section{Contribuciones de los autores}

Preparación y ejecución: HRM, LMC, ARM; Desarrollo de la metodología: HRM, LMC, ARM; Concepción y diseño: HRM, LMC, ARM; Edición del artículo: HRM, LMC, ARM; Supervisión del estudio HRM, LMC, ARM.

\section{LITERATURA CITADA}

- Apaza, V., Cáceres, G., Estrada, R., \& Pinedo, R. (2013). Catálogo de variedades comerciales de quinua en el Perú. Lima: FAO. Disponible en http://www.fao.org/3/a-as890s.pdf

- Bán, R., Virányi, F., \& Komjáti, H. (2004). Benzothiadiaziole-induced resistance to Plasmopara halstedii (Farl.) Berl. et de Toni in Sunflower. En Advances in Downy Mildew Research. 2: 265-274.

- Bazile, D., Jacobsen, S.E., \& Verniau, A. (2016). The Global Expansion of Quinoa: Trends and Limits. Frontiers in Plant Science, 7:1-6. http://doi.org/10.3389/fpls.2016.00622

- Bonifacio, A. (2001). Resitencia de la quinua al mildiu. En Cultivos Andinos. Roma, Italia: FAO. Disponible en http://www.fao.org/tempref/GI/Reserved/FTP FaoRlc/old/prior/segalim/prodalim/prodveg/cdr om/contenido/libro14/cap3.5.htm\#Top

- Choi, Y.-J., Danielsen, S., Lübeck, M., Hong, S.B., Delhey, R., \& Shin, H.D. (2010). Morphological and molecular characterization of the causal agent of downy mildew on Quinoa (Chenopodium quinoa). Mycopathologia, 169(5):403-412. http://doi.org/10.1007/s11046010-9272-y
- Cruces, L. M. (2016). Estrategias en el cultivo de quinua para fortalecer el sistema agroalimentario en la zona andina. Quinua: Manejo Integrado de Plagas. Santiago: FAO. Disponible en http://www.fao.org/publications/card/es/c/0336f c7c-a013-410d-9dec-ee8d0d0438f0/

- Danielsen, S., \& Munk, L. (2004). Evaluation of disease assessment methods in quinoa for their ability to predict yield loss caused by downy mildew. Crop Protection, 23(3): 219-228. http://doi.org/10.1016/j.cropro.2003.08.010

- El-Assiuty, E.M., Famhy, G.M., Taha, E.M., Fahmy, Z.M., Ismael, A.S.M., Abd-Elghamny, W.R., \& Kafsheer, D.A. (2019). Microscopic visualization of Peronospora variabilis Gäum., the cause of quinoa downy mildew in plant tissues at different stages of plant growth. International Journal of Scientific \& Engineering Research, 10(9).

- El-Assiuty, E., Taha, E., Fahmy, Z., \& Fahmy, G. (2019). Histological and Molecular Detections of Peronospora variabilis Gäum Oospores in Seeds of Quinoa) Chenopodium quinoa L.). The Egyptian Journal Of Experimental Biology (Botany), 15(0):1. http://doi.org/10.5455/egyjebb.2019062011515 8

- Estrada, Z.R. (2013). Cultivo de quinua (Chenopodium quinoa Willd) en la región Cusco. Cusco: Insituto Nacional de Innovación Agraria.

- Gabriel, J., Nayra, L., Vargas, A., Magne, J., Angulo, A., La Torre, J., \& Bonifacio, A. (2012). Quinua de valle (Chenopodium quinoa Willd.): fuente valiosa de resistencia genética al mildiu (Perosnopora farinosa). J Selva Andina Res Soc, 3(2):27-44.

- Gómez, L., \& Aguilar, E. (2016). Guia del cultivo de la quinua. Statewide Agricultural Land Use Baseline 2015. Lima: FAO y UNALM.

http://doi.org/10.1017/CBO9781107415324.004

- Gómez, L., \& Eguiluz, A. (2011). Catálogo del Banco de Germoplasma de Quinua (Chenopodium quinoa Willd.). Lima, Perú: Universidad Nacional Agraria La Molina. 
- Gómez-Pando, L. (2015). Quinoa Breeding. En Quinoa: Improvement and Sustainable Production, 1: 87-107.

- Gómez-Pando, L., Aguilar, E., \& IbañezTremolada, M. (2019). Quinoa (Chenopodium quinoa Willd) Breeding. En J. M. Al-Khayri, S. M. Jain, \& D. V. Johnson (Eds.), Advances in Plant Breeding Strategies: Cereals, 259-316.

- INDECOPI. (2009). Norma técnica peruana: Quinua (Chenopodium quinoa Willd.). Requisitos. Recuperado de http://www.conal.gob.ar/CONASE/actas/ctaCO NASE_2013_04Abr18_AnexoII.pdf

- Khalifa, W., \& Thabet, M. (2018). Variation in downy mildew (Peronospora variabilis Gäum) resistance of some quinoa) Chenopodium quinoa Willd.) cultivars under Egyptian conditions. Middle East Journal of Agriculture Research, 7(2): 671-682.

- Kumar, A., Bhargava, A., Shulka, S., Bahadur, H., \& Ohri, D. (2006). Screening of exotic Chenopodium quinoa accessions for downy mildew resistance under mid-Eastern conditions of India. Crop Protection, 25(2006):879-889. http://doi.org/10.1016/j.cropro.2005.11.012

- Marca, S., Espinoza, E., \& Poblete, A. (2015). Multiplicación de semilla de variedades y ecotipos de quinua en valle de Majes-Arequipa. Revista Investigaciones Altoandinas - Journal of High Andean Investigation, 17(3): 355368.http://doi.org/10.18271/ria.2015.147

- Mohamed, A., Hamza, A., \& Derbalah, A. (2016). Recent approaches for controlling downy mildew of cucumber under greenhouse conditions. Plant Protection Science, 52(1):1-9. http://doi.org/10.17221/63/2015-PPS

- Mujica, S., Canahua, A., \& Saravia, R. (2001). Agronomía del cultivo de quinua. En Quinua (Chenopodium quinoa Willd): Ancestral cultivo andino, alimento del presente y futuro. FAO.

- Navruz-Varli, S., \& Sanlier, N. (2016). Nutritional and health benefits of quinoa (Chenopodium quinoa Willd.). Journal of Cereal Science, $\quad$ 69: 371-376. http://doi.org/10.1016/j.jcs.2016.05.004

- Risco, A., Apaza, W., \& Mattos, L.L. (2018). Identificacion del agente causal del mildiu de la albahaca y metodos para la estimacion de su severidad. Anales Científicos, 79(1):159-167. http://doi.org/http://dx.doi.org/10.21704/ac.v79i 1.1158

- Risco, A., \& Mattos, L. (2015). Severidad de Peronospora variabilis Gäum. en Chenopodium quinoa Willd. 'Pasankalla' como respuesta a aplicaciones de fungicidas sintéticos $\mathrm{y}$ bioestimulantes. Anales Científicos, 76(2):382392. http://doi.org/10.21704/ac.v76i2.805

- Romero, C.A. (2015). Quinua Peruana. Situación Actual y Perspectivas en el Mercado Nacional e Internacional al 2015 (Primera ed). Lima, Perú: Dirección de Estudio Económicos e Información. Recuperado de http://repositorio.minagri.gob.pe/handle/MINA GRI $/ 28$

- Romero, C.A. (2017). La quinua: Producción y Comercio del Perú. Lima, Perú: DEEIA (Dirección de Estudios Económicos e Información Agraria).

- Taha, E.M. (2020). Molecular detection and phylogeny of Peronospora variabilis Gäum., the casual agent of downy mildew disease of quinoa at diferent growth stages .Plant Cell Biotechnology and Molecular Biology, (January), 23-24. Disponible en http://www.ikprress.org/index.php/PCBMB/arti cle/view/4842

- Thines, M., \& Choi, Y. (2016). Evolution, Diversity, and Taxonomy of the Peronosporaceae, with Focus on the Genus Peronospora. Phytopathology, 106(1): 6-18. http://doi.org/10.1094/PHYTO-05-15-0127RVW

- Vilcacundo, R., \& Hernández-Ledesma, B. (2017). Nutritional and biological value of quinoa (Chenopodium quinoa Willd.). Current Opinion in Food Science, 14:1-6. http://doi.org/10.1016/j.cofs.2016.11.007. 